\title{
The search for water: Is it so easy to diagnose acute myocarditis?
}

\author{
G. Ross Farris, $M D{ }^{a}{ }^{a}$ and Steven G. Lloyd, $M D, P h D^{a, b}$ \\ a Division of Cardiovascular Disease, Department of Internal Medicine, University of Alabama at \\ Birmingham, Birmingham, AL \\ b Birmingham VA Medical Center, Birmingham, AL
}

Received Dec 19, 2017; accepted Dec 20, 2017

doi: $10.1007 / \mathrm{s} 12350-018-1226-7$

\section{See related article, pp. 857-865}

Acute myocarditis (AM) is a common clinical problem that affects approximately 1.5 million people per year worldwide. ${ }^{1}$ However, the true incidence of AM is unknown and likely underestimated due to low sensitivity of diagnostic methods and low clinical suspicion. The etiologies of AM are varied and include autoimmune, drug reactions, and infection, the most common cause. ${ }^{2,3}$ Diagnosis of AM is difficult to ascertain because patients may range in symptoms from asymptomatic to severe disturbances-including malignant arrhythmias, cardiogenic shock, or even death. Additionally, current World Health Organization (WHO) and International Society and Federation of Cardiology (ISFC) guidelines specify that the diagnosis be made on the grounds of established histologic, immunological, and immunohistochemical criteria. ${ }^{3}$ Because of this, many patients with suspected AM never receive a formal diagnosis. Electrocardiography (ECG), echocardiography, and cardiovascular magnetic resonance (CMR) are all useful noninvasive aids in increasing or decreasing suspicion of AM in a given patient. Endomyocardial biopsy (EMB) is currently considered the gold standard, ${ }^{4}$ but has limited use due to its invasive nature and low sensitivity secondary to sampling errors. ${ }^{5}$ CMR is now being used more

Reprint requests: Steven G. Lloyd, MD, PhD, Division of Cardiovascular Disease, Department of Internal Medicine, University of Alabama at Birmingham, 1808 7th Avenue South, BDB 201, Birmingham, AL 35244; slloyd@uabmc.edu

J Nucl Cardiol 2019;26:866-8.

$1071-3581 / \$ 34.00$

Copyright (C) 2018 American Society of Nuclear Cardiology. frequently as a first-line noninvasive diagnostic tool with good sensitivity and specificity. The current recommended CMR diagnostic criteria are based on T2weighted imaging and two different contrast-enhanced techniques, the early gadolinium enhancement (EGE) and late gadolinium enhancement (LGE) — the so-called “'Lake Louise Criteria', (LLC) requiring at least two of three findings of abnormal T2 signal suggesting myocardial edema, EGE suggesting hyperemia, and LGE suggesting myocardial injury in a nonischemic pattern. ${ }^{6}$

In this issue of JNC, Imbriaco et al. investigate the above criteria, noting the marked variation of sensitivity and negative predictive value in the literature. ${ }^{7}$ They then seek to compare CMR qualitative and qualitative analytical methods for the evaluation of patients with suspected AM, including (after exclusions) 61 patients with suspected AM with CMR within approximately $7 \pm 4$ days of admission. The size of the study is consistent with other work published within this topic. $80 \%$ of the patients studied were finally diagnosed with AM. Troponin and CK levels were significantly higher in the patients with AM, although only $43 \%$ of the patients with AM had an abnormal troponin level. They found no significant differences in diagnostic test performance among qualitative measures-T2- weighted STIR (Short-TI Inversion Recovery), EGE, and LGE sequences, as well as overall LLC (i.e., two of three positive features) for the diagnosis of AM. Among quantitative measures, the Edema ratio (ER, defined as the ratio of signal intensity on T2-weighted STIR images between LV myocardium and pectoralis muscle) and global relative enhancement (RE, defined as the increase in signal intensity in myocardium following contrast infusion, compared to pre-contrast signal intensity) were higher in patients with AM compared to those without. As with the qualitative measures, there were no significant differences in diagnostic test performance among any of 
the quantitative methods in the identification of patients with AM. Because the addition of gadolinium contrastenhanced images did not perform better than the noncontrast, T2-weighted STIR alone, the authors concluded that identification of edema (water) may be the only necessary test in evaluating AM, and that use of contrast may not be necessary.

The authors undertake an important qualitative and quantitative analysis for AM. They build on prior knowledge of utility of MRI in AM, and most importantly their findings may lead to further, larger studies of the relative utility of the various CMR measures of diagnosing AM.

The most important limitation of this study is the lack of validation of CMR findings with pathology, as recognized by the authors. There are limited data comparing CMR to endomyocardial biopsy (EMB), and the authors also acknowledge the drawbacks of EMB. This includes an overall complication rate of $6 \%$ and a serious complication rate of $0.1-0.5 \% .^{8}$ Additionally, sensitivity of EMB is low due to the heterogeneous nature of AM. ${ }^{5}$ As noted in the consensus JACC White Paper, ${ }^{6}$ many CMR studies use clinical diagnosis to validate, which has inherent limitations.

Other potential pitfalls of the study include the fact that CMR was not obtained until around $7 \pm 4$ days after admission for the average patient. While this is in line with the current guidelines, AM improves with time; therefore, a delay in imaging may reduce the sensitivity of the test. With regard to the patient population, ECG abnormalities were only present in $26 \%$ of patients studied, and evident viral syndrome was present in only $25 \%$. Furthermore, the incidence of positive troponin was low for a group with suspected AM (although in patients with troponin elevation, the degree of elevation was quite high compared to their normal range). While several studies considered in formulation of the LLC used clinical diagnosis as the gold standard, ${ }^{9-12}$ these studies were fairly stringent in clinically diagnosing AM, requiring either ECG changes or troponin elevation to enroll, and some required serologic evidence of viral infection that could cause AM or a recent flu-like or diarrheal illness. ${ }^{9,10}$ Imbriaco et al. employed the European Society of Cardiology guidelines for management of acute coronary syndromes in patients presenting without persistent ST-segment elevation to support the diagnosis of $\mathrm{AM},{ }^{13}$ potentially reducing the pre-test probability for AM. Lastly, despite presumably normal coronary angiography, some patients were excluded post angiography due to an ischemic pattern on the CMR LGE imaging. It is unclear whether the authors dismissed these patients because they thought the ischemic pattern was due to vasospasm, embolic MI, or other reasons in the setting of normal coronary arteries.
Myocardial infarction with no obstructive coronary atherosclerosis (MINOCA) is a syndrome defined as evidence of a myocardial infarction (MI) including elevated troponin or other cardiac biomarkers and at least two of ischemic symptoms, new ST or T wave changes, and/or new left bundle branch block and coronary angiography with no epicardial vessel with greater than $50 \%$ stenosis. ${ }^{14}$ The primary differential diagnosis for MINOCA includes AM, Tako-tsubo cardiomyopathy, and coronary artery spasm. Other causes include hypertrophic cardiomyopathy, pericarditis, and amyloidosis, but these are much less frequent. CMR is useful in diagnosing AM and Tako-tsubo cardiomyopathy, but is less useful in diagnosing spasm. While the authors excluded patients with coronary artery disease by angiography, it is unclear if and how the authors excluded spasm as a cause of the abnormalities on CMR, as less prolonged spasm might not result in the characteristic LGE pattern of a Type I myocardial infarction (following a vascular distribution corresponding to that of a major epicardial coronary artery).

The authors suggest that current guidelines (i.e., use of LLC) may need to be revised given the results of this study and suggest that patients may not need to undergo contrast administration as the accuracy of qualitative and quantitative methods is similar. This could expand the application of CMR since a distinct subset of patients may have contraindications to contrast administration. At the very least, omission of the contrast administration would certainly make test performance considerably faster and better tolerated for the patient. Will the current study change practice in diagnosing AM? Probably not at this moment-important technical details needed to implement the clinical CMR protocol are not specified in this paper, including whether a surface coil proximity correction method was applied (critical in understanding the effects of signal intensity changes in pectoralis muscle compared to myocardium), and the specific values for CMR acquisition parameters such as repetition, echo, and inversion times which have major impact on the image signal intensity. However, it is certainly likely that this paper could lead to changes in the priority of imaging acquisition. T2-weighted STIR could be obtained early in the imaging protocol, so that if the imaging session has to be terminated early for any reason, a diagnosis still could be made. And, most importantly, it is quite likely that the findings presented here will lead to further comparative effectiveness research among the various CMR and other techniques for diagnosis of this common and clinically important disorder. Imbriaco et al. have suggested that looking for water (edema) is the way to diagnose AM. Now that we have been led to the water, will we drink? 


\section{Disclosure}

G. Ross Farris and Steven G. Lloyd have no conflicts of interest related to the topic of this manuscript.

\section{References}

1. Global Burden of Disease Study 2013 Collaborators. Global, regional, and national incidence, prevalence, and years lived with disability for 301 acute and chronic diseases and injuries in 188 countries, 1990-2013: a systematic analysis for the Global Burden of Disease Study 2013. Lancet 2015;386(9995):743-800.

2. Cooper LT. Myocarditis. N Engl J Med 2009;360(15):1526-38.

3. Caforio, A.L., et al Current state of knowledge on aetiology, diagnosis, management, and therapy of myocarditis: A position statement of the European Society of Cardiology Working Group on Myocardial and Pericardial Diseases. Eur Heart J 2013;34(33):2636-48, 2648a-2648d.

4. Kindermann I, et al Update on myocarditis. J Am Coll Cardiol 2012;59(9):779-92.

5. Hauck AJ, Kearney DL, Edwards WD. Evaluation of postmortem endomyocardial biopsy specimens from 38 patients with lymphocytic myocarditis: implications for role of sampling error. Mayo Clin Proc 1989;64(10):1235-45.

6. Friedrich $\mathrm{MG}$, et al Cardiovascular magnetic resonance in myocarditis: A JACC White Paper. J Am Coll Cardiol 2009;53(17):1475-87.

7. Imbriaco, M., et al Assessment of acute myocarditis by cardiac magnetic resonance imaging: comparison of qualitative and quantitative analysis methods. U.F. II, Editor. J Nucl Cardiol 2018.
8. Cooper LT, et al The role of endomyocardial biopsy in the management of cardiovascular disease: a scientific statement from the American Heart Association, the American College of Cardiology, and the European Society of Cardiology. Endorsed by the Heart Failure Society of America and the Heart Failure Association of the European Society of Cardiology. J Am Coll Cardiol 2007;50(19):1914-31.

9. Friedrich MG, et al Contrast media-enhanced magnetic resonance imaging visualizes myocardial changes in the course of viral myocarditis. Circulation 1998;97(18):1802-9.

10. Laissy JP, et al MRI of acute myocarditis: A comprehensive approach based on various imaging sequences. Chest 2002;122(5):1638-48.

11. Laissy JP, et al Differentiating acute myocardial infarction from myocarditis: diagnostic value of early- and delayed-perfusion cardiac MR imaging. Radiology 2005;237(1):75-82.

12. Abdel-Aty $\mathrm{H}$, et al Diagnostic performance of cardiovascular magnetic resonance in patients with suspected acute myocarditis: comparison of different approaches. J Am Coll Cardiol 2005;45(11):1815-22.

13. Hamm CW, et al ESC Guidelines for the management of acute coronary syndromes in patients presenting without persistent STsegment elevation: The Task Force for the management of acute coronary syndromes (ACS) in patients presenting without persistent ST-segment elevation of the European Society of Cardiology (ESC). Eur Heart J 2011;32(23):2999-3054.

14. Pasupathy S, et al Systematic review of patients presenting with suspected myocardial infarction and nonobstructive coronary arteries. Circulation 2015;131(10):861-70. 